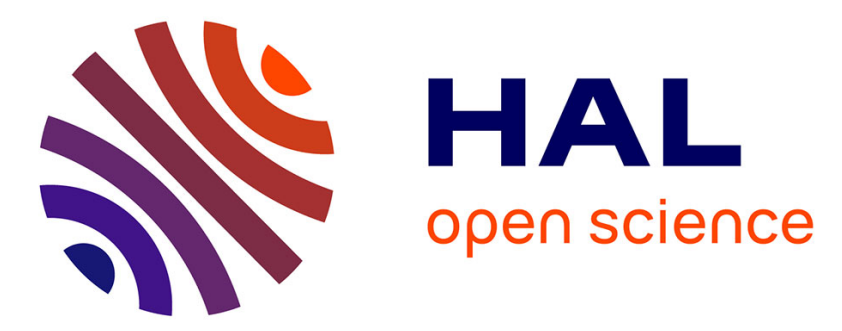

\title{
Descriptive and Intuitive Population-Based Cardiac Motion Analysis via Sparsity Constrained Tensor Decomposition
}

Kristin Mcleod, Maxime Sermesant, Philipp Beerbaum, Xavier Pennec

\section{To cite this version:}

Kristin Mcleod, Maxime Sermesant, Philipp Beerbaum, Xavier Pennec. Descriptive and Intuitive Population-Based Cardiac Motion Analysis via Sparsity Constrained Tensor Decomposition. Medical Image Computing and Computer Assisted Intervention (MICCAI 2015), Oct 2015, Munich, Germany. pp.419-426, 10.1007/978-3-319-24574-4_50 . hal-01205535

\section{HAL Id: hal-01205535 \\ https://hal.inria.fr/hal-01205535}

Submitted on 25 Sep 2015

HAL is a multi-disciplinary open access archive for the deposit and dissemination of scientific research documents, whether they are published or not. The documents may come from teaching and research institutions in France or abroad, or from public or private research centers.
L'archive ouverte pluridisciplinaire HAL, est destinée au dépôt et à la diffusion de documents scientifiques de niveau recherche, publiés ou non, émanant des établissements d'enseignement et de recherche français ou étrangers, des laboratoires publics ou privés. 


\title{
Descriptive and Intuitive Population-Based Cardiac Motion Analysis via Sparsity Constrained Tensor Decomposition
}

\author{
K. McLeod ${ }^{1,2,3}$, M. Sermesant ${ }^{3}$, Philipp Beerbaum, ${ }^{4}$, and X. Pennec ${ }^{3}$ \\ 1 Simula Research Laboratory, Cardiac Modelling Project, Oslo, Norway \\ 2 Centre for Cardiological Innovation, Norway \\ 3 INRIA Méditerranée, Asclepios Project, Sophia Antipolis, France \\ 4 Hanover Medical University, Germany
}

\begin{abstract}
Analysing and understanding population-specific cardiac function is a challenging task due to the complex dynamics observed in both healthy and diseased subjects and the difficulty in quantitatively comparing the motion in different subjects. It was proposed to use affine parameters extracted from a Polyaffine motion model for a group of subjects to represent the $3 \mathrm{D}$ motion regionally over time for a group of subjects. We propose to construct from these parameters a 4-way tensor of the rotation, stretch, shear, and translation components of each affine matrix defined in an intuitive local coordinate system, stacked per region, for each affine component, over time, and for all subjects. From this tensor, Tucker decomposition can be applied with a constraint of sparsity on the core tensor in order to extract a few key, easily interpretable modes for each subject. Using this construction of a data tensor, the tensors of multiple groups can be stacked and collectively decomposed in order to compare and discriminate the motion in each group by analysing the different loadings of each combination of modes for each group. The proposed method was applied to study and compare left ventricular dynamics for a group of healthy adult subjects and a group of adults with repaired Tetralogy of Fallot.
\end{abstract}

\section{Introduction}

Given the challenges in quantitatively measuring cardiac function, beyond simple 1D measures such as volume, strain, ejection fraction and so on, a number of cardiac motion tracking methods have been proposed. Cardiac motion tracking provides a non-invasive means to quantify cardiac motion, and can be used to assess global and regional functional abnormalities such as akinesia or dyskinesia, to classify subjects as healthy/diseased or according to the severity of motion abnormalities, as well as to aid with diagnosis and therapy planning by providing quantitative regional and global measures of function.

While single-subject motion tracking can provide useful insight into the motion dynamics for a given subject, population-based (i.e. atlas-based) motion 
analysis can give further understanding on how the motion dynamics in a patient are typically affected by a pathology. The key challenge with analysing population-wide motion dynamics is in finding a method to be able to compare the motion from one subject to another in a consistent manner. Recent work to address this has been focused on comparing either the regional strain values directly, or the displacements computed from subject-specific motion tracking. Comparing the strain values avoids the need to spatially or temporally align the data, and can provide useful insight to aid with classification, such as in [1]. However, 1D strain measures are sufficient for identifying abnormal regions, but not for fully characterising abnormalities. The displacement fields from a set of subjects can provide further characterisation of motion abnormalities, though this requires spatio-temporal alignment of either the images prior to motion tracking (as in [2]), or the displacements themselves (as in [3],[4]). In either case, spatiotemporal alignment of 3D data is not straightforward and subsequent analysis of the motion from 3D displacements remains difficult to interpret and analyse. In order to address these issues, a method was recently proposed to describe the full motion of a group of subjects in a consistent and low-dimensional manner as the tensor of Polyaffine parameters for a set of regions in the heart over the cardiac cycle [5].

Given that a set of motion parameters is typically high dimensional (due to the need to account for the spatial and temporal factors), model reduction can be useful to reduce the dimensionality of the data while retaining a small number of latent variables that describe the data. Tensor decomposition is one such technique that has been widely studied in the last years for a wide range of applications (see [6] for a review of tensor decomposition methods). PCA of displacement field has already been proposed for population-based cardiac motion analysis in [4], and singular value decomposition (SVD) of stacked Polyaffine motion parameters was proposed in [7]. A difficulty with these methods is in interpreting the results since both PCA and SVD are unconstrained and can thus result in factor matrices with a large number of mode combinations required for each subject.

Inspired by the method developed in [5], we propose a method for populationwide cardiac motion analysis with intelligible and easy to interpret mode combinations. In contrast to this previous work, we study the motion of different population subgroups using descriptive anatomical motion parameters (namely the circumferential twisting, radial thickening, and longitudinal shrinking). Furthermore, we identify much fewer, and thus more easily interpretable, factors discriminating between the motion patterns of healthy and unhealthy subjects thanks to a Tucker decomposition on Polyaffine motion parameters with a constraint on the sparsity of the core tensor (which essentially defines the loadings of each mode combination). We believe that the sparsity of the discriminating factors and their individual intelligibility is the key for a clear and intuitive interpretation of differences between populations. The key contributions of the present work are summarised in the following: 
- Re-orientation of the polyaffine matrices to a prolate spheroidal coordinate system

- Analysis of the rotation, stretch, shear, and translation components

- Combined basis computation of multiple groups

- 4-way tensor decomposition, decoupling the spatial components

- Tucker decomposition performed with sparsity constraints on the core tensor

A brief overview of the methods proposed in [5] is given in the methods section (Sec. 2) to introduce the Polyaffine motion parameter estimation and spatiotemporal alignment of parameters. The key contributions of the present work are then detailed in the subsequent sections. The results obtained from applying the proposed method to distinguish between healthy subjects and patients with Tetralogy of Fallot are described in the results section (Sec. 3).

\section{Methods}

A method for performing population-based cardiac motion analysis methods by considering spatio-temporally aligned Polyaffine motion parameters and performing decomposition of these for a group of subjects was recently proposed in [5]. The method involves a cardiac motion tracking step that takes a dense displacement field computed using the LogDemons algorithm [8] and projects this to a Polyaffine space [9], subject to some cardiac-specific constraints (namely incompressibility and regional smoothing). The obtained Polyaffine parameters are then spatially and temporally aligned to a common reference frame, and the parameters for all subjects are grouped to a data tensor of [space $\times$ time $\times$ subject]. Non-constrained Tucker decomposition is applied to the data tensor to extract the dominant spatial and temporal patterns.

In order to obtain more meaningful parameters and interpretations, we propose in this work to first re-orient the affine parameters from a Cartesian frame to a prolate-spheroidal coordinate system, as described in Sec. 2.1. The rotation, stretch, shear, and translation components can be extracted, as described in Sec. 2.2. We propose to construct a 4 -way tensor to de-couple the spatial components into the affine and regional parts, as described in Sec 2.3, from which Tucker decomposition with a constraint on the sparsity of the core tensor is applied on the 4 -way re-oriented data tensor of each transformation component, as described in Sec. 2.4. Finally, the analysis can be performed by stacking together multiple groups as a single tensor to compute a combined basis, as described in Sec 2.5.

2.1. Re-orientation of Affine Matrices to Local Coordinates: Analysing the affine parameters directly when they are described in Cartesian coordinates creates a difficulty in interpreting differences (or similarities) between groups. In contrast, the parameters can be more easily interpreted once they are in a prolate spheroidal system (which can be computed using the methods described in [11]), given that the parameters will then directly represent motion along the circumferential $(c)$, radial $(r)$, and longitudinal $(l)$ directions. The Jacobian matrices defined at the barycentre of each region in prolate spheroidal coordinates 
$\left(J_{i(P S S)}\right)$ were computed in [5], in order to re-orient the affine parameters to a common reference frame: $M_{i(P S S)}=J_{i(P S S)} * M_{i}$, where $M_{i}$ is the log-affine matrix at region $i$ in Cartesian coordinates, and $M_{i(P S S)}$ is the transformation of $M_{i}$ to prolate spheroidal coordinates.

2.2. Extraction of Rotation, Stretch, Shear, and Translation: Rather than performing the tensor decomposition directly on the parameters, we can perform tensor decomposition on re-scaled rotation, stretch, shearing and translation parameters. In this way, rather than performing the decomposition on the affine matrices $M_{w}^{u}$ :

$$
M_{w}^{u}=\left[\begin{array}{llll}
a_{1,1} & a_{2,1} & a_{3,1} & t_{1} \\
a_{1,2} & a_{2,2} & a_{3,2} & t_{2} \\
a_{1,3} & a_{2,3} & a_{3,3} & t_{3}
\end{array}\right]
$$

the analysis can be performed on vectors $P_{u, v, w}$ made up of the rotation, stretch, shearing and translation components for subject $u$ at time $v$ in region $w$. The components are given by:

$$
\left.\begin{array}{rr}
\text { Rotation }: & {\left[\begin{array}{l}
R_{c} \\
R_{r} \\
R_{l}
\end{array}\right]=\left[\begin{array}{l}
1 / 2\left(a_{2,3}-a_{3,2}\right) \\
1 / 2\left(a_{1,3}-a_{3,1}\right) \\
1 / 2\left(a_{1,2}-a_{2,1}\right)
\end{array}\right]} \\
\text { Shear }: & {\left[\begin{array}{l}
S_{c r} \\
S_{c l} \\
S_{r l}
\end{array}\right]=\left[\begin{array}{l}
1 / 2\left(a_{1,2}+a_{2,1}\right) \\
1 / 2\left(a_{1,3}+a_{3,1}\right) \\
1 / 2\left(a_{2,3}+a_{3,2}\right)
\end{array}\right] \quad \text { Translation }:\left[\begin{array}{l}
S_{c} \\
S_{r} \\
S_{l}
\end{array}\right]=\left[\begin{array}{l}
a_{1,1} \\
a_{2,2} \\
a_{3,3}
\end{array}\right]=\left[\begin{array}{l}
t_{1} \\
t_{2}
\end{array}\right]} \\
t_{3}
\end{array}\right]
$$

Combining the re-scaled affine parameters to a new $[12 \times 1]$ vector, we obtain $P_{u, v, w}=\left[\begin{array}{llllllllllll}R_{c} & R_{r} & R_{l} & S_{c} & S_{r} & S_{l} & S_{c r} & S_{c l} & S_{r l} & T_{c} & T_{r} & T_{l}\end{array}\right]^{T}$. The elements of $P_{u, v, w}$ can then be scaled by the variance of each element described by: $\sigma=\left[\sigma_{R_{c}}, \sigma_{R_{r}} \cdots \sigma_{t_{l}}\right]$, where

$$
\sigma_{[i]}=\sqrt{\frac{1}{N_{u} N_{v} N_{w}} \sum_{u, v, w}\left(p_{u, v, w}\right)_{[i]}^{2}} .
$$

Scaling by the variance ensures that all parameters are equally weighted in the decomposition. Tensor analysis can then be performed on the final vectors $\mathcal{T}_{u, v, w}=\left[P_{u, v, w}\right]_{[i]} / \sigma_{[i]}$.

2.3. 4-Way Tensor Decomposition: The goal of the proposed methodology is to gain insight into pathology-specific functional behaviour by analysing the motion. In order to analyse the spatial motion features independently (in terms of regional and affine components), 4-way Tucker Decomposition can be performed on tensors stacked by [motion parameters $\times$ region $\times$ time $\times$ subject]. Performing decomposition on the full tensor directly has the advantage of describing how all the components interact (as opposed to matricising the tensor and performing 2-way decomposition). Given the complex nature of cardiac 
motion with several key components, trying to analyze these independently is difficult.

The Tucker tensor decomposition method [12] is a higher-order extension of PCA which computes orthonormal subspaces associated with each axis of the tensor $\mathcal{T}$. The Tucker decomposition of an 4 -way tensor $\mathcal{T}$ is expressed as an n-mode product:

$$
\begin{aligned}
\mathcal{T} & \approx \mathcal{G} \times{ }_{1} A_{1} \times_{2} A_{2} \times_{3} A_{3} \times_{4} A_{4} \\
& =\sum_{m_{1}=1}^{M_{1}} \sum_{m_{2}=1}^{M_{2}} \sum_{m_{3}=1}^{M_{3}} \sum_{m_{4}=1}^{M_{4}} g_{m_{1} m_{2} m_{3} m_{4}} a_{1 m_{1}} \otimes a_{2 m_{2}} \otimes a_{3 m_{3}} \otimes a_{4 m_{4}} \\
& =\left[\left[\mathcal{G} ; A_{1}, A_{2}, A_{3}, A_{4}\right]\right],
\end{aligned}
$$

where $\times_{n}$ denotes the mode-n tensor-matrix product, and $\otimes$ denotes the vector outer product. $A_{i}$ are factor matrices in the $i^{\text {th }}$ direction $(i=1 \ldots 4)$ that can be thought of as the tensor equivalent of principal components for each axis. The core tensor $\mathcal{G}$ gives the relationship between the modes in each direction and describes how to re-combine the modes to obtain the original tensor $\mathcal{T}$. We propose to perform 4-way Tucker decomposition with $A_{1}$ : the extracted reoriented affine parameters, $A_{2}$ : the regions, $A_{3}$ : time, and $A_{4}$ : the subject axis.

2.4. Tucker Decomposition with Sparsity Constraints: In order to improve the interpretability of the solution, a sparsity constraint on the core tensor can be incorporated into the Tucker decomposition. Several methods have been proposed for incorporating such a constraint. In [13], an alternating proximal gradient method is used to solve the sparse non-negative Tucker decomposition (NTD) problem:

$$
\begin{gathered}
\min \frac{1}{2}\left\|\mathcal{T}-\mathcal{G} \times{ }_{1} A_{1} \times{ }_{2} A_{2} \times{ }_{3} A_{3} \times{ }_{4} A_{4}\right\|_{F}^{2}+\lambda_{\mathcal{G}}\|\mathcal{G}\|_{1}+\sum_{n=1}^{N} \lambda_{n}\left\|A_{n}\right\|_{1} \\
\text { s.t } \mathcal{G} \in \mathbb{R}_{+}^{J_{1} \times J_{2} \times J_{3} \times J_{4}}, A_{n} \in \mathbb{R}_{+}^{I_{n} \times J_{n}} \forall n,
\end{gathered}
$$

where $J_{n}$ is the dimension of the core tensor for axis $n$ and $\lambda_{\mathcal{G}}, \lambda_{n}$ are parameters controlling the balance of the data fitting and sparsity level. The core tensor $\mathcal{G}$ and factor matrices $A_{n}$ are alternately updated in the order: $\mathcal{G}, A_{1}, \mathcal{G}, A_{2}, \mathcal{G}, A_{3}, \mathcal{G}, A_{4}$ (see [13] for details on the $\lambda$ parameters).

In this work, the tensor $\mathcal{T}$ represents the log-affine parameters and are typically not non-negative. In order to satisfy the non-negativity constraint of the NTD algorithm, the exponential of each element of $\mathcal{T}$ can be analysed rather than $\mathcal{T}$ directly.

2.5. Combined Basis Computation of Multiple Groups: In order to compare the two populations with the same basis, a combined model can be generated by forming a 4 -way tensor of all subjects grouped together, yielding an observation tensor $\mathrm{M}$ of size $[12 \times 17 \times 29 \times N]$ for the affine, regional, temporal, and the $N$ patient-specific components respectively. 4-way tensor decomposition 
can then be applied to this data tensor (as described in the previous sections). By performing the decomposition jointly to obtain a combined basis for multiple groups, the modes relevant to a given population can be extracted by studying the loadings of each patient of a chosen mode to identify mutual and distinct motion patterns.

\section{Experiments and Results}

The proposed methodology was applied to the STACOM 2011 cardiac motion tracking challenge dataset [14]: an openly available data-set of 15 healthy subjects ( 3 female, mean age $\pm \mathrm{SD}=28 \pm 5$ ), as well as a data-set of 10 Tetralogy of Fallot patients ( 5 female, mean age $\pm \mathrm{SD}=21 \pm 7$ ). For all subjects, short axis cine MRI sequences were acquired with 12 - 16 slices, isotropic in-plane resolution ranging from $1.15 \times 1.15 \mathrm{~mm}^{2}$ to $1.36 \times 1.36 \mathrm{~mm}^{2}$, slice thickness of $8 \mathrm{~mm}$, and 15 - 30 image frames.

The sparse NTD algorithm described in Sec. 2 was applied to the stacked parameters for the combined tensor of healthy controls and the Tetralogy of Fallot subjects with the size of the core tensor chosen as $[5 \times 5 \times 5]$ (to retain only the first 5 dominant modes in each axis). The choice of the number of modes is a trade-off between maintaining a sufficient level of accuracy (in terms of the percentage of variance explained), while minimizing the number of output parameters. In this work, 5 modes were considered to be a reasonable trade-off. The core tensor loadings for each subject were averaged for the different groups, in order to visualise the dominant mode combinations in each group. These are plotted in Fig. 1 and indicate that the two groups share some common dominant loadings, though the Tetralogy of Fallot group have some additional dominant loadings, which is expected since additional modes may be required to represent the abnormal motion patterns in these patients.

The dominant modes unique to the control group (indicated by purple arrows in Fig. 1) have the same regional component as the dominant mode in Fig. 2 and the same temporal component (mode 2: black line in Fig. 2 b), along with affine mode 1,3 (see Fig. 2 for description of each matrix element):

$$
A f f_{1}=\left[\begin{array}{cccc}
0 & 0.053 & 0.002 & 0 \\
0.015 & \mathbf{0 . 6 9 7} & 0.009 & 0 \\
0.328 & 0 & 0 & 0.009
\end{array}\right] \quad A f f_{3}=\left[\begin{array}{cccc}
0 & 0.144 & 0.126 & 0 \\
0.099 & 0.101 & 0.076 & 0.083 \\
0 & \mathbf{0 . 8 4 7} & 0 & 0.007
\end{array}\right]
$$

The affine modes suggest dominance in the radial stretch (thickening) and longitudinal rotation (twisting) for mode 1 , and dominance in the longitudinal stretch for mode 3, which are the expected motion dynamics in healthy subjects. The temporal mode (2) accounts for differences around peak systole and diastole (given that the temporal resampling used in this work was linear).

The common dominant mode combinations are plotted in Fig. 2 (top row). The affine mode for the dominant mode combinations (Fig. 2, a) shows predominant stretching in the circumferential direction, which may be related to the 


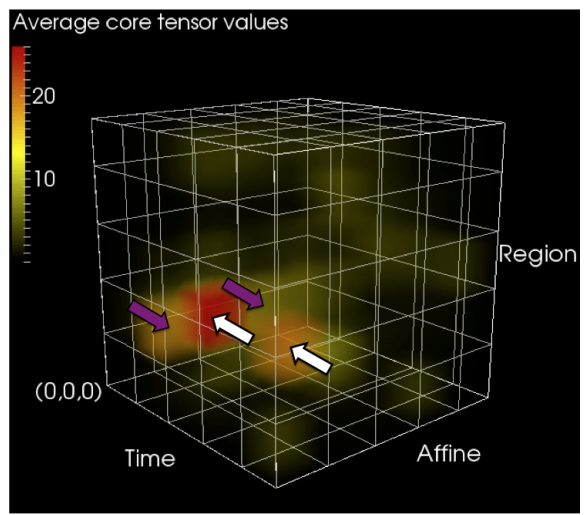

(a) Healthy control group

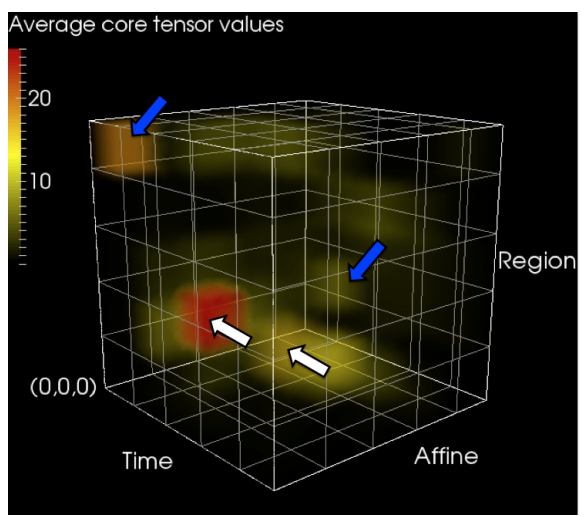

(b) Tetralogy of Fallot group

Fig. 1. Average core tensor loadings for the healthy control group (a) and the Tetralogy of Fallot group (b). The groups share some common dominant loadings (white arrows), however, the control group have some distinct mode combinations (purple arrows). The Tetralogy of Fallot group have some additional extreme values (blue arrows), which indicates that additional modes are needed to represent the abnormal motion patterns in these patients. Arrows indicate the locations of the largest loadings for each group.

twisting motion in the left ventricle. The temporal modes (Fig. 2, b) show a dominant pattern around the end- and mid-diastolic phases for mode 2, which may be due to the end of relaxation and end of filling. The dominant regions for these mode combinations are anterior (Fig. 2, c).

The dominant mode combinations for the Tetralogy of Fallot group are plotted in Fig. 2. The affine mode for the first dominant combination (Fig. 2, d) indicates little longitudinal motion. The corresponding temporal mode (Fig. 2, e) represents a peak at the end systolic frame (around one third of the length of the cardiac cycle). The corresponding regional mode (Fig. 2, f) indicates that there is a dominance in the motion in the lateral wall. This is an area with known motion abnormalities in these patients given that the motion in the free wall of the left ventricle is dragged towards the septum. The temporal mode for the second dominant mode (Fig. 2, h) has instead a peak around mid-systole, with corresponding regional mode (Fig. 2, i), indicating dominance around the apex, which may be due to poor resolution at the apex.

\section{Conclusion}

A method for descriptive and intuitive analysis of cardiac motion in different populations is described. The proposed method makes use of a Polyaffine motion model that represents the motion with reasonable accuracy (i.e. on a par with state-of-the-art methods) while requiring only few, consistently defined parameters for motion tracking of different subjects. The parameters are described 


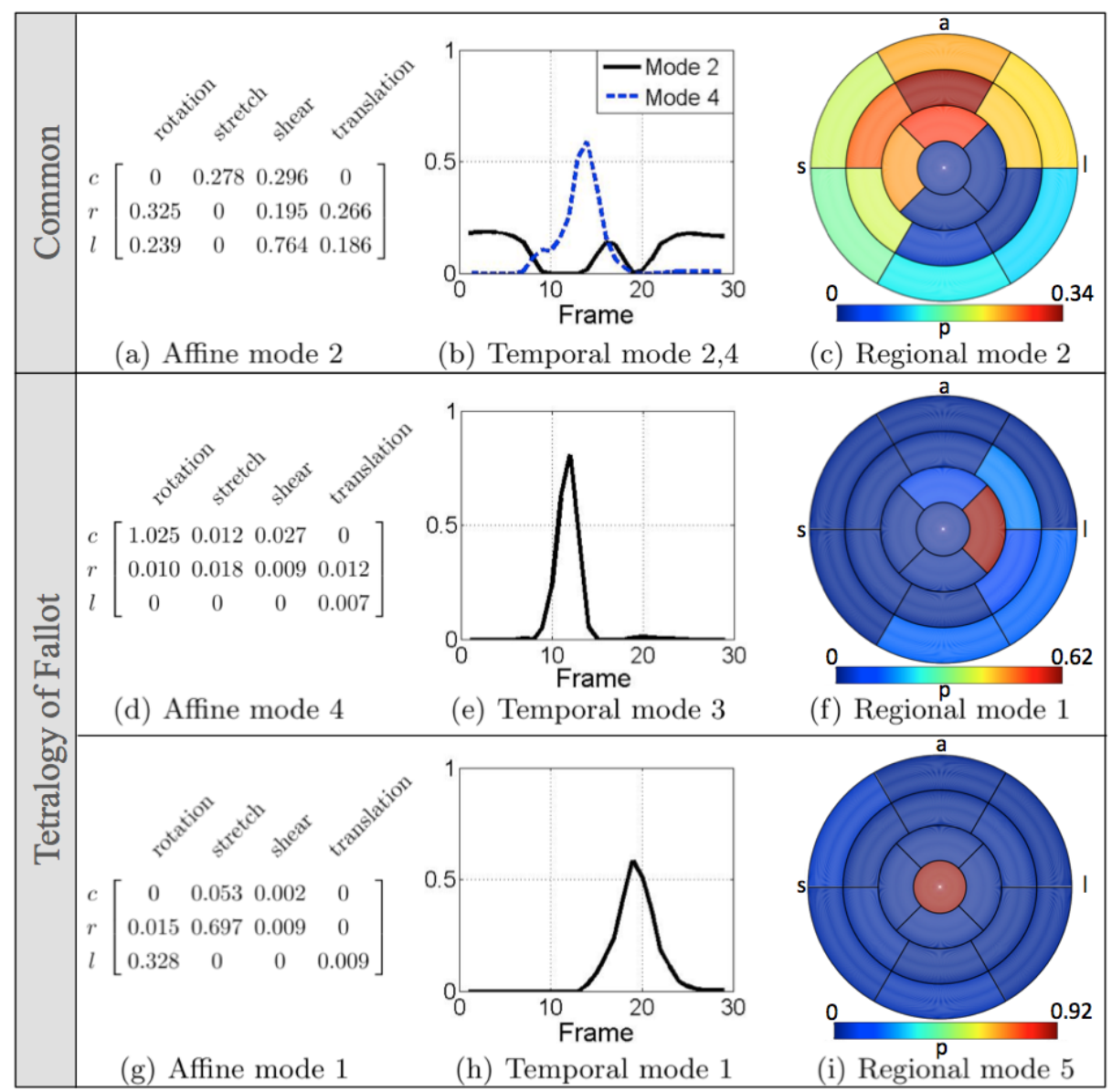

Fig. 2. Dominant mode combinations common to both cohorts: affine mode 2 (a), temporal modes 2 and 4 (b), and regional mode 2 (c). Key - a: anterior, p: posterior, s: septal, l: lateral.

in terms of intuitive physiological parameters and the key affine descriptors of the motion (namely the rotation, stretch, shear, and translation) are analysed collectively for multiple populations in order to determine common and distinct motion patterns between different groups. By performing sparse tensor decomposition of the combined parameters, dominant loadings can be extracted in order to make the analysis and comparison more straightforward, and we believe that obtaining a very small number of expressive and intelligible parameters is crucial for the future automatic discovery of key motion features in different cardiac diseases. The proposed method shows promise for analysing pathology-specific motion patterns in terms of the affine, temporal, and regional factors.

Acknowledgements: This project was partially funded by the Centre for Cardiological Innovation (CCI), Norway funded by the Norwegian Research Council, and the MD Paedigree European project ICT-2011.5.2 grant no. 600932. These tools are largely 
based on the Tensor Toolbox from Sandia National Laboratories. The NTD method used in the work was provided by the authors of [13].

\section{References}

1. Qian, Z., Liu, Q., Metaxas, D.N., Axel, L.: Identifying regional cardiac abnormalities from myocardial strains using nontracking-based strain estimation and spatio-temporal tensor analysis. IEEE Trans. Med. Imaging 30(12) (2011) 20172029

2. Perperidis, D., Rao, A., Lorenzo-Valdés, M., Mohiaddin, R., Rueckert, D.: Spatiotemporal alignment of 4D cardiac MR images. In: Proc. FIMH. Springer (2003) 205-214

3. Rao, A., Sanchez-Ortiz, G.I., Chandrashekara, R., Lorenzo-Valdés, M., Mohiaddin, R., Rueckert, D.: Comparison of cardiac motion across subjects using non-rigid registration. In: Proc. MICCAI. Springer (2002) 722-729

4. Rougon, N.F., Petitjean, C., Preteux, F.J.: Building and using a statistical 3D motion atlas for analyzing myocardial contraction in MRI. In: Med. Imaging, International Society for Optics and Photonics (2004) 253-264

5. McLeod, K., Sermesant, M., Beerbaum, P., Pennec, X.: Spatio-temporal tensor decomposition of a polyaffine motion model for a better analysis of pathological left ventricular dynamics. IEEE Trans. Med. Imaging (2015)

6. Kolda, T.G., Bader, B.W.: Tensor decompositions and applications. SIAM review 51(3) (2009) 455-500

7. Mcleod, K., Seiler, C., Sermesant, M., Pennec, X.: Spatio-temporal dimension reduction of cardiac motion for group-wise analysis and statistical testing. In: Proc. MICCAI, Springer (2013) 501-508

8. Vercauteren, T., Pennec, X., Perchant, A., Ayache, N.: Diffeomorphic demons: Efficient non-parametric image registration. NeuroImage 45(1) (2009) S61-S72

9. Seiler, C., Pennec, X., Reyes, M.: Capturing the multiscale anatomical shape variability with polyaffine transformation trees. Med. Image Anal. (2012)

10. McLeod, K., Seiler, C., Sermesant, M., Pennec, X.: A near-incompressible polyaffine motion model for cardiac function analysis. In: Proc. STACOM MICCAI Workshop, Springer (2012)

11. Toussaint, N., Stoeck, C.T., Sermesant, M., Schaeffter, T., Kozerke, S., Batchelor, P.G.: In vivo human cardiac fibre architecture estimation using shape-based diffusion tensor processing. Med. Image Anal. (2013)

12. Tucker, L.R.: Implications of factor analysis of three-way matrices for measurement of change. Problems in Measuring Change (1963) 122-137

13. Xu, Y.: Alternating proximal gradient method for sparse nonnegative tucker decomposition. Mathematical Programming Computation (2014) 1-32

14. Tobon-Gomez, C., De-Craene, M., et al.: Benchmarking framework for myocardial tracking and deformation algorithms: An open access database. Med. Image Anal. (2013) 